${ }^{1}$ Department Of Psychology, University of Bologna, Cesena, Italy;

${ }^{2}$ Psychologist, Independent Researcher, Savignano, Italy and ${ }^{3}$ Mental Health Department, AUSL ROMAGNA, Cesena, Italy

${ }^{*}$ Corresponding author.

doi: $10.1192 /$ j.eurpsy.2021.373

Introduction: Literature showed that patients suffering from disorders belonging to the schizophrenic (SZ) and bipolar (DB) spectrum have a qualitatively similar but quantitatively different neurocognitive impairment that correlates with the outcomes. However, the majority of former studies are conducted on patients in remission phase.

Objectives: This study aims to compare cognitive functions between SZ and DB in the acute phase and their possible correlations with treatment outcomes.

Methods: In a prospective longitudinal study conducted at the SPDC Ausl unit of Romagna - Cesena, 57 SZ and 82 DB took part in the study. The diagnosis was based on the SCID5 CV and SCID5 DP. Symptom severity was assessed with BPRS and HONOS both at the beginning and at the end of hospitalization. Executive functions were measured with Tower of London (ToL) and Modified Wisconsin Card Sorting Test (MCST), attention with Attentive Matrices (MA) and Stroop Test (ST), non-verbal logic skills with Colored Matrices by Raven (PM47). The statistical analyzes applied are ANOVA and logistic regression.

Results: The cognitive tests did not reveal significant differences between SZ and DB. The logistic regression analysis showed that the scores obtained at the MCST and MA positively correlate with the efficacy of the treatment for both groups.

Conclusions: Cognition in DB and SZ patients was similarly impaired, supporting recent theories that placed diagnoses on a continuum of severity. Moreover, the results indicated that also in the acute phase the best predictors of the outcome were flexibility in problem solving strategies and visuospatial attention.

Disclosure: No significant relationships.

Keywords: Neuropsychological profile; cognitive functions; problem solving; outcomes

\section{Obsessive-compulsive disorder}

\section{8}

The role of mood disorders in the longitudinal course of obsessive-compulsive disorder: Preliminary data from a 20-year prospective follow-up study

\author{
S. Bramante ${ }^{1 \star}$, A. Borsotti ${ }^{1}$, S. Rigardetto ${ }^{2}$ and G. Maina ${ }^{1}$ \\ ${ }^{1}$ Neuroscience, università degli studi di torino, torino, Italy and ${ }^{2} \mathrm{Scdu}$ \\ Psichiatria, Azienda Ospedaliero Universitaria San Luigi Gonzaga, \\ Orbassano, Italy \\ ${ }^{*}$ Corresponding author. \\ doi: 10.1192/j.eurpsy.2021.374
}

Introduction: Although OCD is believed to have a chronic course, little research has been conducted on this, and there are discrepant findings. Studies over the last years have found that a significant proportion of patients with OCD shows symptomatic remission over long term, however there are significant variations in sampling, clinical characteristics, follow-up, and outcome assessments.
Objectives: The present prospective study aims to examine rates of OCD remission after 20 years of follow up and to explore demographic and clinical predictors of remission.

Methods: The study sample consists of adult patients with a principal OCD diagnosis and Y-BOCS total score $\geq 16$, who have been referred to the Department of Neuroscience, University of Turin (Italy). OCD symptoms were assessed every 5 years over the 20 -year follow-up period. Course data were examined using standard survival analysis methods; Cox proportional hazards regression was used to estimate relative hazards for predictors of remission.

Results: There were 360 participants in the study. At year 20, the 28.7 $\%$ of the total sample showed OCD remission. Predictor of remission were female gender, lower Y-BOCS mean scores at study entry, longer duration of illness and comorbidity with major depressive disorder. No specific predictors of full remission were found. Lower Y-BOCS mean scores and comorbid bipolar disorder predicted partial remission.

Conclusions: This study suggests that a significant proportion of patients with OCD shows remission. Future studies are needed to clearly identify predictors of remission.

Disclosure: No significant relationships.

Keywords: obsessive compulsive disorder; follow-up; mood disorders; remission

\section{9}

White matter disconnection and decreased functional connectivity between orbitofrontal cortex and the contralateral temporo-occipital cortex in adults with obsessive compulsive disorder

J. Queiroz ${ }^{1}$, J. Oliveira ${ }^{2}$, A. Maia ${ }^{1}$, C. Fonseca ${ }^{3}$, T. Quendera ${ }^{1}$, A. Oliveira-Maia ${ }^{2,4}$ and B. Barahona-Correa ${ }^{2,4 *}$

${ }^{1}$ Neuropsychiatry Unit, Champalimaud Research, Champalimaud Foundation Centre for the Unknown, Lisbon, Portugal;

${ }^{2}$ Neuropsychiatry Unit, Champalimaud Research and Clinical Centre, Champalimaud Foundation Centre for the Unknown, Lisbon,

Portugal; ${ }^{3}$ Department Of Physics, Faculdade de Ciências da

Universidade de Lisboa, Lisbon, Portugal and ${ }^{4}$ Departamento De

Psiquiatria E Saúde Mental, NOVA Medical School | Faculdade de

Ciências Médicas de Lisboa, Lisbon, Portugal

${ }^{*}$ Corresponding author.

doi: $10.1192 /$ j.eurpsy.2021.375

Introduction: Obsessive compulsive disorder (OCD) affects 2-3\% of the general population. The neurobiology of OCD has been linked to dysfunction of cortico-striatal circuits connecting the orbitofrontal (OFC) to the striatum. Recently, this loop has become an approved target for non-invasive neuromodulatory treatment of OCD.

Objectives: To explore structural and functional connectivity of the OFC in OCD subjects and healthy controls.

Methods: 14 OCD patients and 12 age/sex-matched controls underwent magnetic resonance imaging (MRI) (3T-Philips scanner) for diffusion tensor imaging (DTI) and resting state functional connectivity (rsFC). DTI images were brain extracted and corrected for movement and eddy currents. A diffusion tensor model was fitted to each voxel and used to generate Fractional Anisotropy (FA) maps. Voxel-wise statistical analysis of FA was performed using Tract-Based Spatial Statistics. RsFC images were 